\title{
REAL-TIME MEETING SCHEDULING MODEL BY COMPUTER SUPPORTED COOPERATIVE WORK
}

\author{
Goran Martinović ${ }^{1}$, Leo Budin ${ }^{2}$ \\ ${ }^{\prime}$ Faculty of Electrical Engineering, Josip Juraj Strossmayer University of Osijek, Kneza \\ Trpimira 2b, 31000 Osijek, Croatia (Phone: +38531224766 ; Fax:+38531208905; \\ E-mail:goran.martinovic@etfos.hr); ${ }^{2}$ Faculty of Electrical Engineering and Computing, \\ University of Zagreb, Unska 3, 10000 Zagreb, Croatia (Phone:+38516129936; E-mail: \\ leo.budin@fer.hr)
}

\begin{abstract}
Computer supported cooperative work (CSCW) implies a group of people doing work supported by the computer system and modern networking technologies. The proposed real-time $\mathrm{CSCW}$ meeting scheduling model includes a group, jobs, cooperation and scheduling. Availability, experience, knowledge, interests and the internal infrastructure present group members. Real-time properties of jobs are execution time, deadline, period and interarrival time. The scheduling problem consists of assigning jobs to members of the previously formed groups. The described model represents a prerequisite for harder real-time requests in $\mathrm{CSCW}$.
\end{abstract}

Keywords: Computer Supported Cooperative Work, Human, Meeting, Scheduling

\section{INTRODUCTION}

The importance of knowledge and information in the globalization process, but also the need for cooperation in problem solving increase constantly. Real-time problems are rather extensive. A human represents an active component in CSCW. CSCW combines understanding of the way people work in groups with enabling technologies of computer networking, and associated hardware, software, services and techniques, as in [1] and [2]. 
The paper includes significant organization and timing parameters of $\mathrm{CSCW}$ meeting scheduling. The model is focused on the properties of group members meetings at which they cooperate. Group members possess both knowledge and capabilities. Group effectiveness is mostly determined by the member cooperation, as in [6]. That cooperation has a goal to better understand tasks, allocate resources and develop the cooperative strategy, as in [3].

\section{MEETING SCHEDULING IN CSCW}

Scheduling in the CSCW system can be presented by group $G$, job that has to be done by the group $J$, cooperation enabling carrying out of the job $C$ and scheduling procedure $S$ :

$$
(G, J, C, S)
$$

Group $G$, according to (2) consists of $n$ members:

$$
G=\left\{M_{1}, M_{2}, \ldots, M_{n}\right\}
$$

Every member $M_{i}$ of the group $G$ can be shown as in (3):

$$
M_{i}=\left\{A_{i}, e_{i}, s_{i}, l_{P_{i}}, l_{G i}, D_{i}, I_{i}, C S_{i}\right\} \text {, where: }
$$

$A_{i}$ - member availability according to the schedule

$e_{i}-$ an experience level of a group member

$s_{i}$ - a special knowledge level of a group member

$l_{P i}$ - a personal interest level of a group member

$l_{G i}$ - a general interest level of a group member

$D_{i}$ - the law of statistical distribution of group member's availability

$I_{i}$ - internal ordering of group member's infrastructure

$C S_{i}$ - credits given to a member

Job $J$ that should be carried out by the group consists of $m$ tasks:

$$
J=\left\{T_{1}, T_{2}, \ldots, T_{m}\right\}
$$

Every task can be presented in a general case as in (5). $c_{j}$ is the worst case execution time, $t_{d j}$ is the deadline, $p_{j}$ is the period for a periodic task, $t_{i a}$ is the interarrival time for an aperiodic task and $H / S$ determines a hard or a soft deadline.

$$
T_{j}=\left(c_{j}, t_{d j}, p_{j}, t_{i a j}, H / S\right)
$$

A condition for the group to execute a job is that members must cooperate. The cooperation or meeting is denoted by $C$. Out of the total 
number of meetings $q$, the $k$-th meeting $C_{k}$ can be shown as in (6), where $\mu$ represents organization and infrastructure properties (7), and $\imath$ timing properties.

$$
C_{k}=\left\{\mu_{k}, \tau_{k}\right\}
$$

$\mu_{k}=\left\{G M_{k}, H_{k}, O_{k}, e_{k}, s_{k}, l_{P k}, I_{k}, P_{k}, C S_{k}\right\}$, where

$G M_{k}$ - a set of members participating in the meeting

$H_{k}$ - a set of meeting hosts

$\mathrm{O}_{k}$ - a level of meeting organization

$e_{k}$ - the expected experience level of group members. The experience indicator $e_{i}$ of a future group member (8) must be as in (9).

$$
\begin{aligned}
& \bar{e}_{k}=\sum_{j k=1}^{z} \frac{e_{k}}{z} \\
& e_{i} \geq e_{k}
\end{aligned}
$$

$s_{k}$ is the expected level of special knowledge and must be $s_{i} \geq \bar{s}_{k} . l_{P k}$ is the allowed level of personal interests of members and must be $l_{P i} \leq \bar{l}_{P k} . I_{k}$ is the level of infrastructure ordering of a potential member $I_{i}$. It must be $I_{i} \geq \bar{I}_{k}$. $P_{k}$ is the meeting priority level and $C S_{k}$ is a number of involved champions. Expression (10) shows meeting timing parameters, as in [4].

$\tau_{k}=\left\{t_{c k}, t_{r k}, c_{k}, t_{s k}, t_{d k}, t_{x k}, t_{K k}, p_{k}, t_{i a k}\right\}$, where:

$t_{c k}$ - planned duration of the meeting

$t_{r k}$ - meeting prolongation time

$c_{k}$ - real duration of the meeting, corresponds to sum of $t_{c k}$ and $t_{r k}$

$t_{s k}$ - meeting start time or imagined calendar time of the meeting start

$t_{d k}-$ meeting deadline

$t_{x k}$ - time to decide whether to accept or cancel the meeting

$t_{k k}-$ a relative position of the meeting in the schedule

$p_{k}$ - period of the meeting being carried out

$t_{\text {aik }}$ - interarrival time between consecutive meetings of the same groups

Real-time meeting scheduling by $\mathrm{CSCW}$ is shown in Figure 1. It represents a resource scheduling to users. However, resources represent a job to be done, and the users of these resources are group members. It incorporates the following: definition of job and member parameters, selection of members, scheduler implementation and scheduling according to a relevant parameter, as in [4] and [5]. 


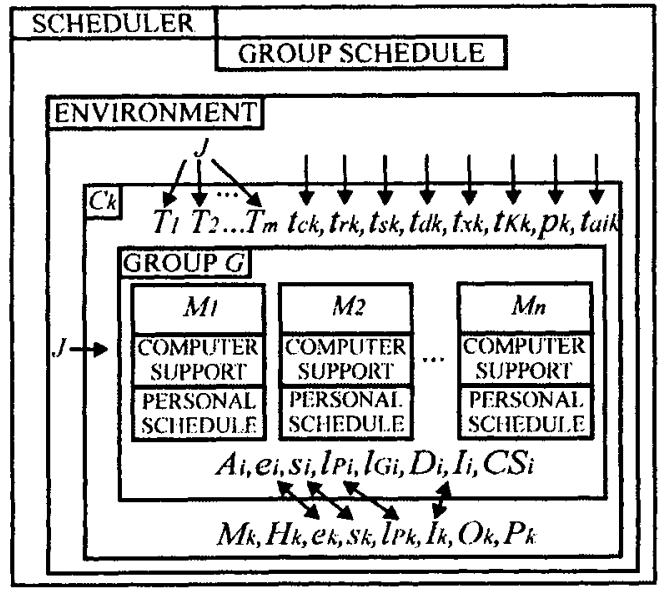

Figure 1. CSCW Meeting Scheduler

\section{CONCLUSION}

The meeting scheduling is presented by the parameters of the group and its members, parameters of jobs that should be executed, properties of cooperation between group members, and the scheduling procedure. Scheduling is based on the cooperation schedule in the calendar format. Special attention is given to a human as a group member. Humans cooperate in problem solving because of their capabilities. The described model represents a precondition for harder real-time requests in CSCW.

\section{REFERENCES}

[1] Borghoff U.M., J.H. Schlichter, Computer Supported Cooperative Work, Introduction to Distributed Applications, Springer - Verlag, 2000.

[2] Greenberg S., E. Chang, Computer Support for Real-Time Collaborative Work, Congressus Numerantium, 75, 1990, pp.247-262.

[3] Li D., L. Zhou, R.R. Muntz, A New Paradigm of User Intention Preservation in Real-Time Collaborative Editing Systems, Proc. of $7^{\text {th }}$ IEEE Int. Conf. on Paralell and Distributed Systems, ICPADS'00, 2000, pp.401-408.

[4] Martinović G., Ž. Hocenski, L. Budin, A Tool for Evaluation of Scheduling Algorithms in Real-Time Systems, $5^{\text {th }}$ WSES/IEEE Int. Conf. CSCC 200, Rethimnon, Crete Island, Greece, 2001, pp. 173-179.

[5] Sandusky R.J., Infrastructure Management as Cooperative Work: Implications for Systems Design, ACM GROUP 97, Phoenix, AZ, USA, 1997, pp.91-100.

[6] Sen S., E. H. Durfee, A Formal Study of Distributed Meeting Scheduling, Proc.Conf. on Organizational Comp. Systems, Atlanta, GA, USA, SIGOIS ACM, 1995, pp. 55-68. 